BULL. AUSTRAL. MATH. SOC.

$08 \mathrm{BI} 5,20 \mathrm{M07}$

VOL. $23(1981), 339-359$.

\title{
VARIETIES OF GROUPS AND OF \\ COMPLETELY SIMPLE SEMIGROUPS
}

\author{
Mario Petrich and Norman R. Reilly
}

\begin{abstract}
Completely simple semigroups form a variety if we consider them both with the multiplication and the operation of inversion. Denote the lattice of all varieties of completely simple semigroups by $L(C S)$ and that of varieties of groups by $L(G)$. We prove that the mappings $V \rightarrow V \cap G$ and $V \rightarrow V \vee G$ are homomorphisms of $L(C S)$ onto $L(G)$ and the interval $[G, C S]$, respectively. The homomorphism $V \rightarrow(V \cap G, V \vee G)$ is an isomorphism of $L(C S)$ onto a subdirect product. We explore different properties of the congruences on $L(C S)$ induced by these homomorphisms.
\end{abstract}

\section{Introduction and summary}

The class of completely simple semigroups is one of the most studied objects in semigroup theory. If considered as a class of universal algebras with the given binary operation and the unary operation of inversion it becomes a variety given by a simple set of identities:

$$
x=x x^{-1} x, x=\left(x^{-1}\right)^{-1}, x x^{-1}=x^{-1} x, x x^{-1}=(x y x)(x y x)^{-1} .
$$

The recent construction of the free completely simple semigroup due to Clifford [1] and Rasin [6] raised the hope that the varieties of completely simple semigroups can be determined via a description of fully invariant congruences on a free completely simple semigroup on a countably infinite set. Indeed, Rasin [6] characterized fully invariant congruences in terms

Received 25 November 1980. 
of certain endomorphisms of the structure group of the free completely simple semigroup.

The present work represents a study of the lattice of varieties of completely simple semigroups by means of two homomorphisms of this lattice:

$$
V \rightarrow V \cap G, \quad V \rightarrow V \vee G,
$$

where $G$ stands for the variety of all groups. We prove that the combination of the two homomorphisms is an isomorphism of the lattice of varieties of completely simple semigroups onto a precisely described subdirect product. Various properties of the above homomorphisms, and the congruences they induce, are discussed in some detail.

Section 2 contains most of the preliminary material needed in the later sections. A characterization of the variety $V \cap G$ is described in Section 3. The homomorphism $V \rightarrow V \cap G$ is discussed in section 4 , and the homomorphism $V \rightarrow V \vee G$ in Section 5. Finally, in Section 6, the homomorphism $V \rightarrow(V \cap G, V \vee G)$ is proved to be an isomorphism onto a subdirect product.

We note that Kleiman [3] has performed an analogous analysis for the lattice of varieties of inverse semigroups. There is a remarkable difference between the case of varieties of inverse semigroups and the varieties of completely simple semigroups: the mapping $V \rightarrow(V \cap G, V \vee G)$ for inverse semigroup varieties is not one-to-one.

\section{Preliminaries}

In general, we use the notation and terminology of Howie [2] or Petrich [5]. In particular, we adopt the notation in [5] for Rees matrix semigroups, and use the description of congruences on a Rees matrix semigroup presented in [2]. In order to minimize the typographical complexity we modify the standard notation for a sandwich matrix and denote the $(j, k)$ th entry by $[j, k]$.

We will consistently use the following notation:

$G$ - the variety of all groups,

$R B$ - the variety of all rectangular bands,

$R G$ - the variety of all rectangular groups (orthodox completely simple), 
CS - the variety of all completely simple semigroups,

$F(G)$ - the lattice of fully invariant subgroups of the group $G$,

$[A, B]$ - the interval of a lattice with minimum $A$ and maximum $B$,

$T_{X}$ - the semigroup of all transformations on a set $X$, $L(V)$ - the lattice of all subvarieties of a variety $V$ of completely simple semigroups,

End $S$ - the semigroup of all endomorphisms of a semigroup $S$.

The first result provides a form for endomorphisms of a Rees matrix semigroup expressed by means of three unique parameters.

LEMMA 2.1 ([6]). Let $S=M(I, G, \Lambda ; P)$, where $P$ is normalized. Let $\varphi \in T_{I}, \omega \in$ End $G, \psi \in T_{\Lambda}$ be such that

(1) $[\lambda, i] \omega=[1 \psi, 1 \varphi][\lambda \psi, 1 \varphi]^{-1}[\lambda \psi, i \varphi][1 \psi, i \varphi]^{-1}(\lambda \in \Lambda, i \in I)$.

Then $\theta=\theta(\varphi, \omega, \psi)$ defined by

$$
(i, g, \lambda) \theta=\left(i \varphi,[1 \psi, i \varphi]^{-1}(g \omega)[1 \psi, 1 \varphi][\lambda \psi, 1 \varphi]^{-1}, \lambda \psi\right)
$$

is an endomorphism of $S$. Conversely, every endomorphism of $S$ can be so written uniquely.

A construction of the Rees matrix representation of a free completely simple semigroup follows.

LEMMA 2.2 ([1], [6]). Let $X=\left\{x_{i} \mid i \in I\right\}$ be a nonempty set, fix

$I \in I$ and let $I^{\prime}=I \backslash\{1\}$. Let

$$
z=\left\{q_{i} \mid i \in I\right\} \cup\left\{[j, k] \mid j, k \in I^{\prime}\right\},
$$

$F_{Z}$ be the free group on $z$, and let $P=([j, k])$ with $[1, k]=[j, 1]=1$, the identity of $F_{Z}$. Then

$$
F=M\left(I, F_{Z}, I ; P\right)
$$

is a free completely simple semigroup over $X$, with embedding $x_{i} \rightarrow\left(i, q_{i}, i\right)$. 
NOTATION 2.3. We fix a countably infinite set $X$, and in addition to the above notation, introduce

$$
F_{q}=\left\langle q_{i} \mid i \in I\right\rangle, F_{p}=\left\langle[j, k] \mid j, k \in I^{\prime}\right\rangle,
$$

the free subgroups of $F_{Z}$ generated by the sets $\left\{q_{i} \mid i \in I\right\}$ and $\left\{[j, k] \mid j, k \in I^{\prime}\right\}$, respectively. We will consistently use the notation $F=M\left(I, F_{Z}, I ; P\right)$ introduced above.

Note that $F_{Z}=F_{q} * F_{p}$, the free product of $F_{q}$ and $F_{p}$. As a consequence of Lemma 2.1, we have

COROLLARY 2.4. If $\theta(\varphi, \omega, \psi\rangle$ is an endomorphism of $F$, then $E_{p} \omega \subseteq F_{p}$

LEMMA 2.5 ([6]). Any fulzy invariant congruence on $F$ is either

(i) idempotent separating or

(ii) a left group congruence or

(iii) a right group congruence or

(iv) a group congruence.

We will need only fully invariant idempotent separating congruences, for they are precisely the ones which correspond to the varieties in the interval [RB, CS]. In this context, the following special case of ([2], Lemma 4.19) is of particular interest.

LEMMA 2.6. Let $S=M(I, G, \Lambda ; P)$. If $N$ is a normal subgroup of $G$, then $\rho_{N}$ defined on $S$ by

$$
(i, g, \lambda) \rho_{N}(j, h, \mu) \Leftrightarrow i=j, g h^{-1} \in N, \lambda=\mu,
$$

is an idempotent separating congmence on $S$, and every such congruence is obtained in this way. Writing $P / N$ for the $\Lambda \times I$ matrix with the $(j, k)$ th entry equal to the $(j, k)$ th entry of $P$ modulo $N, S / p$ is isomorphic to $M(I, G / N, I ; P / N)$.

NOTATION 2.7. We will consistently use the notation $\rho_{N}$ introduced above. For a variety $V$ of completely simple semigroups, we denote by $\rho(V)$ the fully invariant congruence on $F$ corresponding to $V$. Also let 
$E\left(F_{Z}\right)=\left\{\omega \in\right.$ End $F_{Z} \mid$ there exist $\varphi, \psi \in T_{I}$ such that (1) holds $\}$, $E\left(F_{p}\right)=\left\{\omega \in\right.$ End $F_{p} \mid$ there exist $\varphi, \psi \in T_{I}$ such that (1) holds $\}$.

Hence $E\left(F_{Z}\right)$ consists precisely of endomorphisms of $F_{Z}$ that arise in association with endomorphisms of $F$. The latter are uniquely determined by the functions $\left\{q_{i} \mid i \in I\right\} \rightarrow F_{Z}, \varphi, \psi \in T_{I}$ independently. Furthermore, $E\left(F_{p}\right)$ consists precisely of endomorphisms of $F_{p}$ that extend to elements of $E\left(F_{Z}\right)$.

LEMMA 2.8 ([6]). Let $N$ be a normal subgroup of $F_{Z}$. Then $\rho_{N}$ is fully invariant if and only if $N \omega \subseteq N$ for alz $\omega \in E\left(F_{Z}\right)$.

DEFINITION 2.9. A normal subgroup of $F_{z}$ (respectively, $F_{p}$ ) is E-invariant if it is invariant under all $\omega \in E\left(F_{2}\right)$ (respectively, $E\left(F_{p}\right)$ ). The set of all E-invariant subgroups of $F_{Z}$ (respectively, $F_{p}$ ) will be denoted by $N$ (respectively, $N_{p}$ ). For any $N \in N$, let

$$
N_{q}=N \cap F_{q}, N_{p}=N \cap F_{p} \text {. }
$$

It is clear that $N$ (respectively, $N_{p}$ ) is a sublattice of the lattice of all normal subgroups of $F_{Z}$ (respectively $F_{p}$ ), and that each element of $N_{p}$ is the intersection with $F_{p}$ of an element of $N$ (for example, its normal closure in $F_{Z}$ ).

PROPOSITION 2.10 ([6]). The interval [RB, CS] is a complete modular lattice anti-isomorphic to the lattice $N$.

We take advantage of the basic results on varieties of groups as found in [4]. In particular, we recall that the lattice of group varieties is anti-isomorphic to the lattice of fully invariant subgroups of the free group $F_{X}$ on a countable number of generators $X$.

NOTATION 2.11. If $U$ is a group variety corresponding to the fully invariant subgroup $N$ of $F_{X}$ and $G$ is any group, then the smallest normal subgroup $H$ of $G$ for which $G / H \in U$ will be denoted by $N(G)$ or $u(G)$. 


\section{A characterization of $V \cap G$}

We prove here some basic statements which will be used in later sections. In particular, we determine the fully invariant subgroup of $F_{q}$ which corresponds to the variety $V \cap G$.

LEMMA 3.1. Let $N \in N$.

(i) $N_{q}$ is a fully invariant subgroup of $F_{q}$.

Let $u$ be the corresponding group variety, so that $u\left(F_{q}\right)=N_{q}$.

(ii) $u\left(F_{2}\right) \subseteq N$

(iii) $u\left(F_{p}\right) \subseteq N_{p}$.

Proof. (i) Any mapping of the free generators of $F_{Z}$ into $F_{Z}$ extends uniquely to an endomorphism of $F_{Z}$, and conversely, every endomorphism of $F_{Z}$ is uniquely determined by its action on the free generators of $F_{Z}$. Condition (1) for membership in $E\left(F_{Z}\right)$ relates only to the free generators of $F_{p}$ and is trivially satisfied if we choose $\varphi$ and $\psi$ to be the identity mappings.

It follows that any mapping of the free generators of $F_{q}$ into $F_{q}$ extends to an element of $E\left(F_{Z}\right)$. Consequently, any endomorphism of $F_{q}$ extends to an element of $E\left(F_{Z}\right)$. Hence, $N_{q}$ must be invariant under any endomorphism of $F_{q}$ and is thus fully invariant in $F_{q}$.

(ii), (iii) In the same way, any mapping of the free generators of $F_{q}$ into $F_{Z}$ (or $F_{p}$ ) extends to an element of $E\left(E_{Z}\right)$. In particular, there exist $k, \omega \in E\left(F_{Z}\right)$ which restrict to bijections of the free generators of $F_{q}$ onto those of $F_{Z}$ and $F_{p}$, respectively.

The hypothesis $N \in N$ implies

$$
N_{q} \kappa \subseteq N, \quad N_{q} \omega \subseteq N \cap F_{p}=N_{p} .
$$

The restrictions of $\kappa$ and $\omega$ to $F_{q}$ are isomorphisms of $F_{q}$ onto $F_{Z}$ 
and $F_{p}$, respectively, and thus

$$
\begin{aligned}
& u\left(F_{Z}\right)=U\left(F_{q}\right) \kappa=N_{q} \kappa \subseteq N, \\
& u\left(F_{p}\right)=U\left(F_{q}\right) \omega=N_{q} \omega \subseteq N_{p},
\end{aligned}
$$

which completes the proof.

NOTATION 3.2. Let $V \in[R B, C S]$ and $\rho(V)=\rho_{N}$. Then $N_{q}=N \cap F_{q}$ is a fully invariant subgroup of $F_{q}$ and so determines a variety of groups, which we denote by $V_{G}$.

We are now ready for the characterization theorem.

THEOREM 3.3. If $V \in[R B, C S]$ and $\rho(V)=\rho_{N}$, then $V_{G}=V \cap G$.

Proof. The free group on a countable number of generators in $V_{G}$ is simply $F_{q} / N_{q}$. Clearly, $E_{q} / N_{q} \in V \cap G$ and so $V_{G} \subseteq V \cap G$.

For the converse containment, let $H$ be any group in $V \cap G$. Let $\left\{h_{i} \mid i \in I\right\}$ be any countable subset of $H$. Now $\left\{N_{q} q_{i} \mid i \in I\right\}$ is a set of relatively free generators of the relatively free group $E_{q} / N_{q}$. If we can show that there exists a homomorphism $\varphi$ of $E_{q} / N_{q}$ into $H$ such that $\left(N_{q} q_{i}\right) \varphi=h_{i}$, for all $i \in I$, then we shall have, by the arbitrariness of $H$ and the $h_{i}$, that every countably generated subgroup of any element of $V \cap G$ is a homomorphic image of $F_{q} / N_{q}$ and therefore must satisfy all the laws of $V_{G}$. Hence, $V \cap G$ satisfies the laws of $V_{G}$ and so $V \cap G \subseteq V_{G}$, as required.

We will show that such a homomorphism $\varphi$ exists.

We start with the homomorphism $\theta$ of $F$ into $H$ defined on the generators by: $\left(i, q_{i}, i\right) \theta=h_{i}$. Since $(1,1,1)$ is an idempotent, we must have $(1,1,1) \theta=1$. Hence

$$
\begin{aligned}
\left(1, q_{i}, 1\right) \theta & =\left[(1,1,1)\left(i, q_{i}, i\right)(1,1,1)\right] \theta \\
& =\left(i, q_{i}, i\right) \theta=h_{i} .
\end{aligned}
$$


For ease of reference, let

$$
F_{11}=\left\{(1, g, 1) \in F \mid g \in F_{Z}\right\} .
$$

Since $H \in V$, we have $\theta \circ \theta^{-1} \supseteq \rho_{N}$, which implies that the homomorphism $\theta$ factors uniquely through $F / \rho_{N}$. Hence $\theta=\mu \psi$, where $\mu$ is the natural homomorphism of $F$ onto $F / \rho_{N}$ and $\psi$ is a homomorphism of $F / \rho_{N}$ into $H$. We illustrate this situation by the diagram

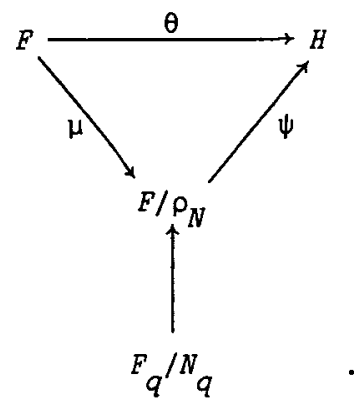

The homomorphism $\psi$ is such that, for all $i \in I$,

$$
\left(1, q_{i}, 1\right) \rho_{N} \psi=\left(1, q_{i}, 1\right) \mu \psi=\left(1, q_{i}, 1\right) \theta=h_{i} \text {. }
$$

However, the mapping

$$
\xi: N_{q} a \rightarrow(1, a, 1) \rho_{N}
$$

of $F_{q} / N_{q}$ into $F_{11} / \rho_{N}=F_{11} \mu$ is a monomorphism. Let $\varphi=\xi \psi$. Then $\varphi$ is a homomorphism of $F_{q} / N_{q}$ into $H$ such that

$$
\left(N_{q} q_{i}\right) \varphi=\left(1, q_{i}, 1\right) p_{N} \psi=h_{i} .
$$

Thus $\varphi$ is the required homomorphism.

\section{The projection of $L(C S)$ onto $L(G)$}

We explore here the relationship between varieties of completely simple semigroups and varieties of groups by considering the projection of $L(C S)$ onto $L(G)$ given by $V \rightarrow V \cap G$. First we introduce two mappings which will prove to be elements of $E\left(F_{2}\right)$ and will play an important role in our discussion. Recall that $F_{2}=F_{q} * F_{p}$. 
NOTATION 4.1. Let $\pi_{q}$ and $\pi_{p}$ be the projections of $F_{2}$ onto $F_{q}$ and $F_{p}$, respectively.

The next result summarizes the most salient features of these projections.

LEMMA 4.2. (i) $\pi_{q}, \pi_{p} \in E\left(F_{Z}\right)$.

(ii) If $N \in N$, then $N \pi_{q}=N_{q}, N \pi_{p}=N_{p}$.

(iii) $\pi_{q}$ induces a lattice homomorphism of $N$ onto $F\left(F_{q}\right)$

Proof. (i) It suffices to produce mappings $\varphi, \psi: I \rightarrow I$ in each case so that condition (1) is satisfied. For $\pi_{q}$ take $i \varphi=i \psi=1$ for all $i \in I$, and for $\pi_{p}$ let $\varphi=\psi$ be the identity mapping on $I$. (In addition, one has $q_{i} \pi_{q}=q_{i}$ and $q_{i} \pi_{p}=1$, for all $i \in I$.)

(ii) Let $N \in N$. Then $N \pi_{q} \subseteq N$ since $\pi_{q} \in E\left(F_{Z}\right)$, and $N \pi_{q} \subseteq F_{q}$ by the definition of $\pi_{q}$, so that $N \pi_{q} \subseteq N \cap F_{q}$. On the other hand, since $N_{q} \subseteq F_{q}$, we have $N_{q}=N_{q} \pi_{q} \subseteq N_{q}$, and thus $N_{q}=N_{q}$. The same type of argument can be used to prove that $N \pi_{p}=N_{p}$.

(iii) Let $M, N \in N$. By part (ii) and Lemma 3.1 (i), we have $N \pi_{q}=N_{q}$ which is a fully invariant subgroup of $F_{q}$; thus $\pi_{q}: N \rightarrow F\left(F_{q}\right)$. In addition,

$$
\begin{aligned}
(M \cap N) \pi_{q} & =(M \cap N)_{q}=M \cap N \cap F_{q}=\left(M \cap F_{q}\right) \cap\left(N \cap F_{q}\right) \\
& =M \pi_{q} \cap N \pi_{q}, \\
(M \vee N) \pi_{q} & =(M N) \pi_{q}=\left(M \pi_{q}\right)\left(N \pi_{q}\right)=M \pi_{q} \vee N \pi_{q},
\end{aligned}
$$

and thus $\pi_{q}$ determines a lattice homomorphism of $N$ into $F\left(F_{q}\right)$. Since $F_{q}$ is a free group on a countable number of generators, any $N \in F\left(F_{q}\right)$ determines a variety of groups $U$, say. Then $M=U\left(F_{Z}\right) \in N$ and $M \pi_{q}=N$. Consequently, the homomorphism induced by $\pi_{q}$ maps $N$ onto $F\left(E_{q}\right)$. 
NOTATION 4.3. For any $v \in L(C S)$, let

$\bar{V}=\{S \in \mathcal{C} S \mid$ all subgroups of $S$ are in $V\}$.

It is readily verified that $\bar{V}$ is a variety of completely simple semigroups. We are now ready for the principal result of this section.

THEOREM 4.4. The mapping

$$
X: V \rightarrow V \cap G \quad(V \in L(C S))
$$

is a homomorphism of $L(C S)$ onto $L(G)$. Denote by a the congruence induced by $x$. For any $v \in L(C S)$, we have

$$
v_{\alpha}=[V \cap G, \bar{V}] \text {. }
$$

Proof. Let $V \in[R B, C S]$ be determined by the fully invariant congruence $\rho_{N}$ on $F$. By Theorem 3.3, the variety of groups determined by the fully invariant subgroup $N_{q}$ of $F_{q}$ is just $V \cap G$. Combining the mappings

$$
V \rightarrow \rho_{N}+N \rightarrow N_{q} \rightarrow V \cap G
$$

we obtain, by Lemma 4.2 (iii), a homomorphism of $[R B, C S]$ onto $L(G)$. It is then straightforward to verify that this homomorphism extends to a homomorphism of $L(C S)$ onto $L(G)$.

The statement concerning $V_{\alpha}$ needs no formal argument.

The rest of the section is devoted to characterizations of the maxima of $\alpha$-classes in terms of identities and subgroups of $F_{Z}$. In the context of completely simple semigroups, group identities are written in the form $u=v$ with $u \neq l \neq v$. In the interest of simplicity, we will frequently abbreviate expressions of the form $u\left(x_{1}, \ldots, x_{n}\right)$ for words in the variables $x_{i}$ to $u\left(x_{i}\right)$. However, for an identity $u\left(x_{1}, \ldots, x_{n}\right)=v\left(x_{1}, \ldots, x_{n}\right)$, it need not be the case that all variables appear on both sides of the identity.

LEMMA 4.5. Let $u\left(x_{1}, \ldots, x_{n}\right)=v\left(x_{1}, \ldots, x_{n}\right)$ be a group identity and $S$ be a completely simple semigroup. Then all subgroups of $S$ satisfy $u\left(x_{i}\right)=v\left(x_{i}\right)$ if and only if $s$ satisfies $u\left(\bar{x}_{i}\right)=v\left(\bar{x}_{i}\right)$, where 
$\bar{x}_{i}=\left(x_{1} x_{1}^{-1}\right) x_{i}\left(x_{1} x_{1}^{-1}\right), \quad 1 \leq i \leq n$.

Proof. Assume that all subgroups of $S$ satisfy the identity $u\left(x_{i}\right)=v\left(x_{i}\right)$. If we assign any value to the variables $x_{1}, x_{2}, \ldots, x_{n}$, all the variables $\bar{x}_{i}$ will be contained in the maximal subgroup of $S$ containing $x_{1}$. Since $u\left(x_{i}\right)=v\left(x_{i}\right)$ is valid in that subgroup, $u\left(\bar{x}_{i}\right)=v\left(\bar{x}_{i}\right)$ is valid in $S$.

Conversely, assume that $S$ satisfies the identity $u\left(\bar{x}_{i}\right)=v\left(\bar{x}_{i}\right)$. If all variables $x_{1}, x_{2}, \ldots, x_{n}$ assume values in the same subgroup $G$ of $S$, then $\bar{x}_{i}=x_{i}$ for $1 \leq i \leq n$, and $G$ satisfies the identity $u\left(x_{i}\right)=v\left(x_{i}\right)$.

COROLLARY 4.6. If $u$ is a variety of groups given by the set of identities $\left\{u_{\alpha}\left(x_{i}\right)=v_{\alpha}\left(x_{i}\right)\right\}_{\alpha \in A}$, then $\bar{u}$ is determined by the system of identities $\left\{u_{\alpha}\left(\bar{x}_{i}\right)=v_{\alpha}\left(\bar{x}_{i}\right)\right\}_{\alpha \in A}$, where $\bar{x}_{i}=\left(x x^{-1}\right) x_{i}\left(x x^{-1}\right)$ and $x$ is a fixed variable.

NOTATION 4.7. For any $S \in C S$, let $\langle S\rangle$ denote the subvariety of CS generated by $S$.

PROPOSITION 4.8. For any $u \in L(G)$, we have $\bar{u}=\left\langle F / \rho_{M}\right\rangle$ where $M=U\left(F_{Z}\right)$.

Proof. First note that $M \in N$ and that $M_{q}=U\left(F_{q}\right)$ is the fully invariant subgroup of $F_{q}$ corresponding to $U$. Theorem 3.3 then gives that $\left(F / \rho_{M}\right) \cap G=U$. Let $V \in L(C S)$ be such that $V \cap G=U$. If $V$ is a variety of groups, left or right groups, then clearly $V \subseteq\left(F / \rho_{M}\right)$. Otherwise, let $U$ be determined by the fully invariant congruence $\rho_{N}$ on $F$. Since $v_{X}=U$, we must have $M=U\left(F_{Z}\right) \subseteq N$. But then $\rho_{M} \subseteq \rho_{N}$ and thus $V \subseteq\left(F / \rho_{M}\right)$. By the maximality of $\bar{u}$, the result follows.

Indeed, we see from Proposition 4.8 that 


$$
F / \rho_{M} \cong M\left(I, F_{Z} / M, I ; P / M\right)
$$

is a relatively free object in $\bar{u}$.

COROLLARY 4.9. Let $v \in[R B, C S]$ and $\rho(V)=\rho_{N}$. Then $v$ is the maximum element of its $\alpha$-class if and only if $N$ is a fully invariant subgroup of $F_{Z}$.

Proof. That $N$ is fully invariant if $V$ is the maximum element of its $\alpha$-class follows immediately from Proposition 4.8. For the converse, suppose that $N$ is fully invariant and let $\rho(\bar{V})=\rho_{M}$. By Proposition 4.8, $M$ is also fully invariant so that, by Theorem 3.3, we have

$$
\left(F_{q} / N_{q}\right\rangle=V \cap G=\bar{V} \cap G=\left(F_{q} / M_{q}\right) .
$$

Thus, in the notation of 2.11 ,

$$
N\left(F_{q}\right)=N_{q}=M_{q}=M\left(F_{q}\right)
$$

from which it follows that $N=M$ and $V=\bar{V}$, as required.

COROLLARY 4.10. The varieties that are maximum (respectively, minimum) in their a-classes form a sublattice of L(CS).

Proof. The varieties that are minimum in their $\alpha$-classes are simply the group varieties and so constitute a sublattice. By Corollary 4.9, the maximum elements correspond to the fully invariant subgroups of $F_{Z}$. Since these form a sublattice of $N$, it follows that the maximum elements form a sublattice of $L(C S)$.

\section{The projection of $L(C S)$ onto $[G, C S]$}

We now turn to the study of the relationship of the lattice $L(C S)$ and its interval $[G, C S]$ via the homomorphism $V \rightarrow V \vee G$. We then characterize the maximal elements of the congruence on $L(C S)$ induced by this homomorphism in two different ways.

NOTATION 5.1. Let $\hat{F}_{p}$ denote the normal closure in $F_{Z}$ of $F_{p}$. For any $N \in N$, let $N_{p}^{*}=N \cap \hat{F}_{p}$ and let

$$
N_{p}^{*}=\left\{N_{p}^{*} \mid N \in N\right\}
$$


The following lemma supplies the necessary information for the main result of this section.

LEMMA 5.2. (i) $N_{p}^{*} \subseteq N$.

(ii) $\rho(R G)=\rho_{\hat{F}_{p}}$.

(iii) $E_{Z}=F_{q} \hat{F}_{p}$.

(iv) $F_{q} \cap \hat{F}_{p}=\{I\}$.

(v) For any $N \in N$, we have $N=N_{q} N_{p}^{*}$.

Proof. (i) Since any endomorphism in $E\left(F_{Z}\right)$ maps $F_{p}$ into $F_{p}$, it must also map $\hat{F}_{p}$ into itself. But then it must map $N \cap \hat{F}_{p}$ into itself, for any $N \in N$.

(ii) For $N=\hat{F}_{p}$, it is clear that $F / \rho_{N}$ is a rectangular group. On the other hand, if $N \in N$ is such that $F / \rho_{N}$ is a rectangular group, then $F_{p} \subseteq N$, and since $N$ is normal, we have $\hat{F}_{p} \subseteq N$.

(iii) This is a consequence of the fact that $F_{2}=F_{q} * F_{p}$.

(iv) Consider the projection $\pi_{q}: F_{Z} \rightarrow F_{q}$. Its kernel is $\hat{F}_{p}$ and it maps $F_{q}$ identically, whence the assertion.

(v) Let $N \in N$ and $n \in N$. By part (iii), $n=a b$ for some $a \in F_{q}, b \in \hat{E}_{p}$. Hence

$$
a=(a b) \pi_{q} \in N \cap F_{q}=N_{q}
$$

so that

$$
b=a^{-1}(a b) \in N \cap \hat{F}_{p}=N_{p}^{*}
$$

Thus $N \subseteq N_{q} N_{p}^{*}$, and the opposite inclusion is trivial.

We first deduce two interesting consequences.

COROLLARY 5.3. For $V \in[R B, C S]$ with $\rho(V)=\rho_{N}$, we have 
$\rho(V \vee G)=\rho_{N_{p}^{*}} . \quad$ Consequently, $R G \subseteq V$ if and only if $N \subseteq \hat{F}_{p}$.

Proof. The hypothesis $V \supseteq R B$ yields

$$
V \vee G=V \vee R B \vee G=V \vee R G \text {, }
$$

whence, by Lemma 5.2 (ii), we get

$$
\begin{aligned}
\rho(V \vee G) & =\rho(V \vee R G)=\rho(V) \cap \rho(R G) \\
& =\rho_{N} \cap \rho_{\hat{F}_{p}}=\rho_{N \cap \hat{F}_{p}}=\rho_{N_{p}^{*}} .
\end{aligned}
$$

COROLLARY 5.4. For $U, v \in[R B, C S]$ with $\rho(U)=\rho_{M}$ and $\rho(V)=\rho_{N}$, we have

$$
U \vee G=V \vee G \Leftrightarrow M_{p}^{*}=N_{p}^{*} .
$$

We are now ready for the principal result of this section.

THEOREM 5.5. The mapping

$$
\theta: V \rightarrow V \vee G \quad(V \in L(C S))
$$

is a homomorphism of $L(C S)$ onto $[G, C S]$.

Proof. We first consider $\theta$ on the interval [RB, CS]. Recall that this interval is anti-isomorphic to $N$. In the light of Corollary 5.3, for any $V \in[R B, C S]$ with $\rho(V)=\rho_{N}$, we have $\rho(V \vee G)=\rho_{N_{p}^{*}}$. Hence it suffices here to show that the mapping

$$
\mu: N+N_{p}^{*}=N \cap \hat{F}_{p}
$$

is a homomorphism of $N$ onto $N_{p}^{*}$.

Obviously $\mu$ maps $N$ into $N_{p}^{*}$ and preserves meets. The onto part is a consequence of Lemma 5.2 (i). It remains to show that for any $M, N \in N$, we have

$$
(M \vee N)_{\mu}=M \mu \vee N \mu,
$$

that is, $(M N)_{p}^{*}=M_{p}^{*} N_{p}^{*}$.

Let $a \in(M N)_{p}^{*}$, say $a=m n$, where $m \in M, n \in N$. By Lemma

5.2 (v), we have $m=m_{1} m_{2}$ and $n=n_{1} n_{2}$ with $m_{1}, n_{1} \in F_{q}$, 
$m_{2}, n_{2} \in \hat{F}_{p}$. Then

$$
m=m_{1} m_{2} n_{1} n_{2}=\left(m_{1} m_{2} m_{1}^{-1}\right)\left[\left(m_{1} n_{1}\right) n_{2}\left(m_{1} n_{1}\right)^{-1}\right] m_{1} n_{1} .
$$

Note that in (2), $m_{2}, n_{2} \in \hat{\vec{F}}_{p}$, which is normal, and also $m=a \in(M N)_{p}^{*}$, which implies that $m_{1} n_{1} \in \hat{F}_{p}$. But then, by Lemma 5.2 (iv), we get $m_{1} n_{1} \in F_{q} \cap \hat{F}_{p}=\{1\}$. Consequently,

$$
a=m=\left(m_{1} m_{2} m_{1}^{-1}\right) n_{2} \in M_{p}^{*} N_{p}^{*}
$$

This proves that $(M N)_{p}^{*} \subseteq M_{p}^{*} N_{p}^{*}$; the opposite inclusion is obvious.

Therefore $\theta$ is a homomorphism on the interval [RB, CS].

To see that $\theta$ is a homomorphism on the entire lattice $L(C S)$, we consider $u \in L(R G)$ and $V \in L(C S)$. It is straightforward to verify that the following is true:

$$
\begin{aligned}
(U \cap V) \vee G & =\{S \in C S \mid S \cong G \times R, G \in G, R \in(U \cap V) \cap R B\} \\
& =(U \vee G) \cap(U \vee G) .
\end{aligned}
$$

Therefore $\theta$ is indeed a homomorphism of $L(C S)$ onto $[G, C S]$.

We have seen in Theorem 4.4 that the congruence $\alpha$ induced by the homomorphism $V \rightarrow V \cap G$ has the property that its classes are intervals of $L(C S)$. We conjecture that the congruence $B$ induced on $L(C S)$ by the homomorphism $V \rightarrow V \vee G$ also has this property. We are unable to prove the existence of the least element of the B-class containing an arbitrary variety $V$, but observe that the greatest element is obviously $V \vee G$. In Corollary 5.3, we have already characterized the corresponding element of $N$. We now turn to the description of $V \vee G$ in terms of the system of identities it satisfies.

NOTATION 5.6. In any group $G$, denote by $x^{\alpha}$ the conjugate $a^{-1} x a$ of $x$. For $i_{t} \neq 1, j_{t} \neq 1, q_{k} \in F_{q}$,

$$
v=v\left(\left[i_{1}, j_{1}\right]^{f_{1}\left(q_{1}, \ldots, q_{n}\right)}, \ldots,\left[i_{m}, j_{m}\right]^{f_{m}\left(q_{1}, \ldots, q_{n}\right)}\right) \in \hat{F}_{p},
$$

let 
354

Mario Petrich and Norman R. Reilly

$$
\hat{v}=v\left(\begin{array}{c}
f_{1}\left(\bar{x}_{1}, \ldots, \bar{x}_{n}\right) \\
z_{i_{1}, j_{1}}
\end{array}, \ldots, z_{i_{m}, j_{m}}^{f_{m}}\left(\bar{x}_{1}, \ldots, \bar{x}_{n}\right)\right)
$$

where .

$$
\begin{aligned}
& \text { (5) } \bar{x}_{k}=x x^{-1} x_{k} x x^{-1}, z_{i_{t}, j_{t}}=\left(x y_{i_{t}}\right)\left(x y_{i_{t}}\right)^{-1}\left(y_{j_{t}} x\right)\left(y_{j_{t}} x\right)^{-1} \\
& \text { for variables } x, x_{k}, y_{i_{t}}, y_{j_{t}}(k=1,2, \ldots, n, t=1,2, \ldots, m) .
\end{aligned}
$$

LEMMA 5.7. Let $V \in[R B, C S], \rho(V)=\rho_{N} ;$ then

$$
N_{p}^{*}=\left\{v \in \hat{F}_{p} \mid \hat{v}^{2}=\hat{v} \text { is a law in } v\right\} \text {. }
$$

Proof. Let $v \in N_{p}^{*}$ be given by (3). Consider any substitution of the variables in $\hat{v}$, see (4), into $F$ :

$$
x \rightarrow a, x_{k} \rightarrow a_{k}, y_{i_{t}} \rightarrow b_{\alpha_{t}}, y_{j_{t}} \rightarrow b_{\beta_{t}} .
$$

Let $\hat{v}^{\sigma}, z_{i_{t}, j_{t}}^{\sigma}$ and so on, denote the elements obtained from $\hat{v}, z_{i_{t}, j_{t}}$ and so on, see (5), by making these substitutions. Without loss of generality, we may assume that $b_{\alpha_{t}} \in H_{-\alpha_{t}}, b_{\beta_{t}} \in H_{\beta_{t^{-}}}$, where $H_{-\alpha_{t}}, H_{B_{t^{-}}}$need not all be distinct even for distinct $\alpha_{t}$ or $\beta_{t}$.

Let $a \in H_{r s}$ and note that

$$
\xi:(r, h, s) \rightarrow[s, r] h \quad\left(h \in F_{Z}\right)
$$

is an isomorphism of $H_{r s}$ onto $F_{Z}$. Then

$$
\bar{x}_{k}^{\sigma} \in H_{r \varepsilon}, \quad g_{k}=\bar{x}_{k}^{\sigma} \xi \in F_{Z},
$$

where the latter part of (6) defines $g_{k}$, and

$$
\begin{aligned}
z_{i_{t}, j_{t}}^{\sigma} & =\left(a b_{\alpha_{t}}\right)\left(a b_{\alpha_{t}}\right)^{-1}\left(b_{\beta_{t}} a\right)\left(b_{\beta_{t}} a\right)^{-1} \\
& =\left(r,\left[\alpha_{t}, r\right]^{-1}, \alpha_{t}\right)\left(\beta_{t},\left[s, \beta_{t}\right]^{-1}, s\right) \\
& =\left(r,\left[\alpha_{t}, r\right]^{-1}\left[\alpha_{t}, \beta_{t}\right]\left[s, \beta_{t}\right]^{-1}, s\right)
\end{aligned}
$$

https://doi.org/10.1017/S0004972700007231 Published online by Cambridge University Press 
Varieties of groups

355

so that

$$
z_{i_{t}, j_{t}}^{\sigma} \xi=[s, r]\left[\alpha_{t}, r\right]^{-1}\left[\alpha_{t}, \beta_{t}\right]\left[s, \beta_{t}\right]^{-1}
$$

Taking into account (6) and (7), we obtain

(8) $\hat{v}^{\sigma} \xi=\left\{\left\{[s, r]\left[\alpha_{1}, r\right]^{-1}\left[\alpha_{1}, \beta_{1}\right]\left[s, \beta_{1}\right]\right\}^{f_{1}\left(g_{1}, \ldots, g_{n}\right)}\right.$

$$
\left.\ldots\left\{[s, r]\left[\alpha_{m}, r\right]^{-1}\left[\alpha_{m}, \beta_{m}\right]\left[s, \beta_{m}\right]\right\}^{f_{m}\left(g_{1}, \ldots, g_{n}\right)}\right) .
$$

Let $\omega \in E\left(F_{Z}\right)$ be defined by (1) and

$$
q_{k} \omega=g_{k}, \quad i_{t} \psi=\alpha_{t}, j_{t} \varphi=\beta_{t}, I \psi=r, I \varphi=s,
$$

and let $\psi$ and $\varphi$ be defined arbitrarily elsewhere. Using (1), we obtain

$$
\begin{aligned}
{\left[i_{t}, j_{t}\right]_{t}^{f_{t}\left(q_{1}, \ldots, q_{n}\right)_{\omega}} } & =\left(\left[i_{t}, j_{t}\right] \omega\right) f_{t}\left(q_{1} \omega, \ldots, q_{n} \omega\right) \\
& =\left([\varepsilon, r]\left[\alpha_{t}, r\right]^{-1}\left[\alpha_{t}, \beta_{t}\right]\left[\varepsilon, \beta_{t}\right]^{-1}\right)^{f_{t}\left(g_{1}, \ldots, g_{n}\right)} .
\end{aligned}
$$

Comparing this with $(8)$, we conclude that $\hat{v}^{\sigma} \xi=v \omega$, where $v \omega \in N_{p}^{*}$ since $v \in N_{p}^{*}$ and $N_{p}^{*} \in N$, by Lemma $5.2(i)$. Since

$$
[s, r]^{-1}(v \omega)^{2}\left([s, r]^{-1}(v \omega)\right)^{-1}=[s, r]^{-1}(v \omega)[s, r] \in N_{p}^{*},
$$

Lemma 2.6 yields

$$
\left(r,[s, r]^{-1}(\nu \omega)^{2}, s\right) \rho_{N_{p}^{*}}\left(r,[\delta, r]^{-1}(v \omega), s\right) .
$$

Using $N_{p}^{*} \subseteq N$ and the definition of $\xi$, we deduce $\left(\hat{v}^{\sigma}\right)^{2} \rho_{N} \hat{v}^{\sigma}$ and thus $\hat{v}^{2}=\hat{v}$ is a law in $V$.

Conversely, suppose that $\hat{v}^{2}=\hat{v}$ is a law in $v$. Consider the substitution

$$
\begin{aligned}
& \sigma: x \rightarrow(1,1,1), x_{i}+\left(1, q_{i}, 1\right), \\
& y_{i_{t}} \rightarrow\left(i_{t}, 1, i_{t}\right), y_{j_{t}}+\left(j_{t}, 1, j_{t}\right) .
\end{aligned}
$$

https://doi.org/10.1017/S0004972700007231 Published online by Cambridge University Press 
Then

$$
\bar{x}_{i}^{\sigma}=\left(1, q_{i}, 1\right), z_{i_{t}, j_{t}}=\left(1,\left|i_{t}, j_{t}\right|, 1\right)
$$

and thus $\hat{v}^{\sigma}=(1, v, 1)$. Since $\hat{v}^{2}=\hat{v}$ is a law in $v$, we get $(1, v, 1)^{2} \rho_{N}(1, v, 1)$ and so $N v^{2}=N v$. But then $v \in N \cap \hat{F}_{p}=N_{p}^{*}$, as required.

PROPOSITION 5.8. Let $V \in[R B, C S], \rho(V)=\rho_{N}$. Then $\left\{\hat{v}^{2}=\hat{v} \mid v \in N_{p}^{*}\right\}$ is a basis of laws for $V \vee G$.

Proof. This is immediate from Corollary 5.4 and Lemma 5.7.

It is a simple consequence of Proposition 5.8 that

$$
F / \rho_{N_{p}^{*}} \cong M\left(I, F_{Z} / N_{p}^{*}, I ; P / N_{p}^{*}\right)
$$

is a relatively free object in $V \vee G$.

\section{Embedding of $L(C S)$ into a subdirect product}

We combine here the homomorphism $X$ of Theorem 4.4 with the homomorphism $\theta$ of Theorem 5.5 and prove that the resulting mapping $V \rightarrow(V \cap G, V \vee G)$ is actually an isomorphism of $L(C S)$ onto a subdirect product of $L(G)$ and $[G, C S]$.

THEOREM. 6.1. The mapping

$$
\xi: V \rightarrow(V \cap G, V \vee G) \quad(V \in L(C S))
$$

is an isomorphism of $L(C S)$ onto the subdirect product of $L(G)$ and $[G, C S]$ consisting of the pairs $(U, v)$ such that $v \subseteq \bar{u} \vee G$. Moreover, for $w \in L(C S)$,

$$
w \cap G=u, \quad w \vee G=v \Leftrightarrow w=\bar{u} \cap v .
$$

Proof. Since $X$ and $\theta$ are homomorphisms (Theorems 4.4 and 5.5) so also is $\xi$. Let $V, w \in[R B, C S]$ and $\rho(V)=\rho_{M}, \rho(w)=\rho_{N}$. Then, by Corollary 5.3,

$$
\rho(V \vee G)=\rho_{M_{p}^{*}}, \quad \rho(W \vee G)=\rho_{N_{p}^{*}}
$$


On the other hand, by Theorem 3.3,

$$
V \cap G=\left\langle F_{q} / M_{q}\right\rangle, W \cap G=\left\langle F_{q} / N_{q}\right\rangle .
$$

If $V \xi=W \xi$, then from (9) and (10), we have

$$
M_{q}=N_{q}, M_{p}^{*}=N_{p}^{*},
$$

and from Lemma $5.2(v)$ it follows that

$$
M=M_{q p}^{M_{p}^{*}}=N_{q p} N_{p}^{*}=N .
$$

Therefore $V=W$ and $\xi$ is one-to-one on [RB, CS].

If either of $V$ or $W$ does not contain $R B$, then it must be a variety of left groups or a variety of right groups (or a variety of groups) and a simple case-by-case argument will again show that $V \xi=\omega \xi$ implies that $V=W$. Therefore $\xi$ is an isomorphism.

For $W \vee L(C S)$, let $u=W \cap G$ and $v=w \vee G$. Then $w \subseteq \bar{u}$ and so $v=\omega \vee G \subseteq \bar{u} \vee G$.

Conversely, let $(U, v) \in L(G) \times[G, C S]$ with $V \subseteq \bar{U} \vee G$, and let $\omega=\bar{u} \cap v$. We have

$$
w \cap G=\bar{u} \cap V \cap G=\bar{u} \cap G=u .
$$

Now consider $W \vee G$. Then

$$
w \vee G=(\bar{u} \cap v) \vee G \subseteq v
$$

since $G \subseteq V$. For the opposite inclusion, first assume that $R G \subseteq V$. Then clearly $R B \subseteq W$. Let $\rho(\bar{U})=\rho_{M}$ and $\rho(V)=\rho_{N}$; also let $m \in M$, $n \in N$ be such that $m \in \in \hat{F}_{p}$ so that $m n \in(M N)_{p}^{*}$. The hypothesis $R G \subseteq V$ implies that $n \in N \subseteq \hat{F}_{p}$, and $V \subseteq \bar{U} \vee G$ implies that

$$
\begin{aligned}
& \rho_{M_{p}^{*}}=\rho_{M \cap \hat{F}_{p}}=\rho(\bar{U} \vee G) \subseteq \rho_{N} \text { so that } M_{p}^{*} \subseteq N \text {. Consequently }
\end{aligned}
$$

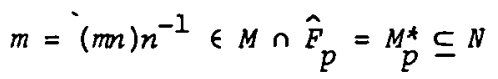

and thus $m n \in N$, which proves that $(M N)_{p}^{*} \subseteq N$. But then

$$
\rho(W \vee G)=\rho(W \vee R G)=\rho_{M N \cap \hat{F}_{p}}=\rho_{(M N)_{p}^{*}} \subseteq \rho_{N}=\rho(V)
$$

and hence $V \subseteq W \vee G$. 
If $V$ does not contain $R G$, then we must have $V$ equal to $G$, or to the variety of left groups or the variety of right groups. Particular, but simpler, arguments will show that $W \vee G=V$ in each of these cases.

We have thus established the converse part of the implication in the statement of the theorem. This establishes that $\xi$ maps $L(C S)$ onto the sublattice $\{(u, v) \mid u \in L(G), G \subseteq V \subseteq \bar{u} \vee G\}$.

The direct part of the implication follows from the fact that $\xi$ is one-to-one.

COROLLARY 6.2. For any $V \in L(C S)$, we have

$$
U=\overline{V \cap G} \cap(U \vee G) \text {. }
$$

REMARK 6.3. By Theorem 3.3 and Corollary 5.3, we have the following associations for any $V \in L(C S)$ :

$$
\begin{aligned}
& x_{q}^{N}=N \cap F_{q} \rightarrow V \cap G \\
& N_{p}^{*}=N \cap \hat{F}_{p} \rightarrow U \vee G .
\end{aligned}
$$

\section{References}

[1] A.H. Clifford, "The free completely regular semigroup on a set", $J$. Algebra 59 (1979), 434-451.

[2] J.M. Howie, An introduction to semigroup theory (London Mathematical Society Monographs, 7. Academic Press, London, New York, San Francisco, 1976).

[3] Е.И. Нлейман [E.I. KleĬman], "Неноторые сзойства струнтуры многообразий ннверсных полугругп" [Some properties of the lattice of varieties of inverse semigroups], Ural. Gos Univ. Mat. Zap. 10 (1977), no. 3 Issied. po Sovremen. Algebre, 56-72, 216-217. "Замечание н статье Е.И. Нлеймана 'Неноторые свойства струнтуры многообразий ннеерсных полугрупп'" [Correction to Some properties of the lattice of varieties of inverse semigroups], Ural. Gos. Univ. Mat. Zap. 11 (1979), 207. 
[4] Hanna Neumann, Varieties of groups (Ergebnisse der Mathematik und ihrer Grenzgebiete, 37. Springer-Verlag, Berlin, Heidelberg, New York, 1967).

[5] Mario Petrich, Introduction to semigroups (Charles E. Merrill, Columbus, Ohio, 1973).

[6] В.В. Раснн [V.V. Rasin], "Свободные вполне простые полугруппы" [Free completely simple semigroups], Ural. Gos. Univ. Mat. Zap. 11 (1979), no. 3, 140-151.

Department of Mathematics,

Simon Fraser University,

Burnaby,

British Columbia V5A IS6,

Canada. 